\title{
Classification of Land Cover in Satellite Image using Supervised and Unsupervised Techniques
}

\author{
Balamurugan $\mathrm{G}$. \\ Assistant Professor, \\ Department of Computer \\ Science and Engineering, \\ Christ College of Engineering and Technology, \\ Pondicherry University, \\ Pondicherry, India
}

\author{
K. B. Jayarraman, $\mathrm{PhD}$ \\ Professor and Head of the Department \\ Department of Computer \\ Science and Engineering, \\ Manakula Vinayagar \\ Institute of Technology, \\ Pondicherry University, \\ Pondicherry, India
}

\begin{abstract}
Remote Sensing plays a vital role for the detection of urban expansion. Due to high complexity of urban landscapes such as building area, vegetation area are classified based on the feature extraction from the satellite Images. Different feature Extraction methods are employed for obtaining the primitives such as texture, shapes and sizes etc. In this paper, obtaining first order statistics, GLCM and Wavelet transformation for the feature extraction and then final classification is processed using proposed supervised and unsupervised Technique for the urban landscape classification.
\end{abstract}

\section{Keywords}

Feature Extraction, First order statistics, GLCM, Wavelet transformation, supervised and unsupervised Technique

\section{INTRODUCTION}

Land coverage Mapping and Change Detection analysis in remote sensing images have been improved because of its vast applications. Remote sensing represents to Images captured from a satellite, where a single satellite image covers a wide area of landscapes which provides lot of information. Therefore to obtain the information from satellite Imagery, efficient algorithms are needed. In spite of high complexities in satellite images for classification, various conventional methods are employed for the classification of landscape coverage. This paper describes the schematic method using wavelet transform employed for the land cover classification. In the proposed method, the texture property is obtained by applying the wavelet transform and wavelet decomposition. Feature Extraction carried out in various classes of land cover images and the performance are measured.

\section{PROPOSED METHODOLOGY}

In proposed method, the first level feature extraction method by first order statistics and the second level feature extraction by GLCM (gray-level co-occurrence matrix) and first order statistics with wavelet transform for various decomposition levels were performed.

In the last level process, the extracted features are processed to supervised and unsupervised classifier and thus obtained the classified output image.

\section{PROCESSING STAGE}

The test satellite image data is preprocessed by converting into gray-scale from color images. The noise is removed by using the best median filter and then the contrast of the image is then enhanced by clearing the resolution of the input image. It made easier for classification by the preprocessing stage. Original image, after the preprocessing operations were segmented from which the first level and second level features were extracted. The feature extraction process was carried out based on pixel and texture analysis. In this paper feature extraction involves first- order statistics and GLCM as the first and second level feature extraction with wavelet transform. The results are obtained and then it has been applied to the proposed supervised and unsupervised classifier technique.

\section{FEATURE EXTRACTION}

The first level feature extractor indicates extraction of features using first-order statistics based techniques. First-order statistics method (FOS)- Based on One dimensional probability density function on the occurrence of pixels between pair of gray level were calculated as the technical features. The preprocessed test data were converted to histogram. The features such as Standard Deviation, Mean, Skewness, and Entropy were computed from the histogram.

$$
\begin{gathered}
\text { Mean } \bar{x}=\frac{1}{N} \sum_{i=1}^{n} \sum_{j=0}^{n} \mathrm{xi} . \mathrm{j} \\
\text { Standard deviation } \sigma=\sqrt{\frac{1}{N}} \sum_{i=0}^{n}\left(x i-h^{2}\right) \\
\text { Skewness }=\frac{\sum_{i=0}^{n}(\mathrm{Xi}-\mathrm{x})^{3}}{(N-1) \sigma^{2}} \\
\text { Entropy }=\sum_{i=1}^{N} \sum_{j=0}^{n}(\mathrm{C}(\mathrm{i}, \mathrm{j})) \log (\mathrm{c}(\mathrm{i}, \mathrm{j}))
\end{gathered}
$$

\section{A. Second Order Statistics Using GLCM Technique}

The second level feature extraction process is carried out with the help of wavelet transformation in combination of gray level co-occurrence matrix. In this the feature extraction from first order statistics is decomposed to form second order statistics features using GLCM and WTFOS. 


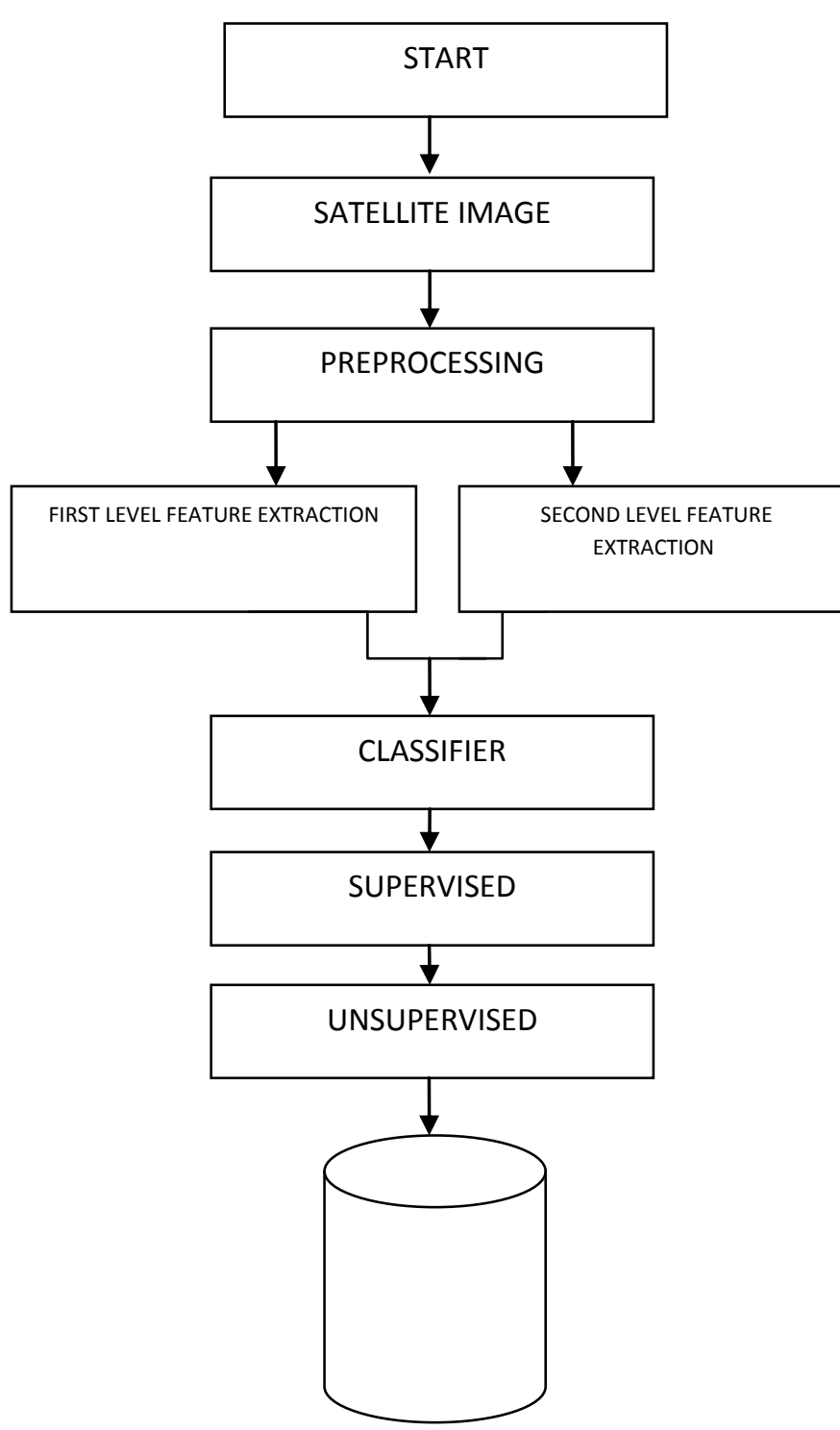

Figure 1 System Architecture

\section{B. Functionalities Of Glcm}

Gray -level Co occurrence Matrix is defined as the distribution of co-occurring values at a given offset over an image. GLCM textures progress the relation between reference and the neighbor pixels at a time. It involves a set of features such as contrast, correlation and entropy.

$$
\begin{gathered}
\text { Contrast }=\sum_{i . j=0}^{n}(\mathrm{i}-\mathrm{j})^{2} \mathrm{C}(\mathrm{i}, \mathrm{j}) \\
\text { Correlation } \mathrm{r}=\frac{1}{N-1} \sum_{\mathrm{x}, \mathrm{y}}^{\mathrm{n}}\left(\frac{\mathrm{x}-\mathrm{x} 1}{\sigma \mathrm{x}}\right) \quad\left(\frac{\mathrm{y}-\mathrm{y} 1}{\sigma \mathrm{y}}\right) \\
\text { Energy }=\sum_{i=0}^{n} \sum_{k=0}^{n}\left(\mathrm{C}(\mathrm{i}, \mathrm{j})^{2}\right)
\end{gathered}
$$

\section{Wavelet Transformation}

Segmentation of remotely sensed Images is predefined as an approach of multi resolution wavelet transform for the textural feature extraction. Multi resolution analysis is carried out with the help of wavelet transformation. Wavelet transformation is processed with the help of orthogonal or biorthogonal mirror filters that supports down sampling. It consists of pair of half-band low-pass and high pass filters which are used as bi-orthogonal mirror filters. Image consists of low frequency components in both horizontal and vertical directions. It decomposed into four sub images level by level denoted as LL $(m, n), \operatorname{LH}(m, n), H L(m, n)$ and $\mathrm{HH}(m, n)$. Here LL $(m, n)$ denoted as low frequency components of the original image, it possess most relevant information of the test satellite image. $\mathrm{LH}(\mathrm{m}, \mathrm{n}), \mathrm{HL}(\mathrm{m}, \mathrm{n})$ and $\mathrm{HH}(\mathrm{m}, \mathrm{n})$ represent Horizontal, vertical and diagonal high frequency components of the test image.

Considered features are average, energy and entropy. The features which processed by energy parameter indicates textual uniformity. The next feature extraction indicates entropy process which measures the disorder present in image. In preprocessing stage $20 \mathrm{X} 20$ window size images is extracted from the input image. The sample 20X20 extracted images is decomposed into four sub images using the two-dimensional wavelet transform. The textual parameters are derived with the help of statistical measure and extracted from decomposed image.

The wavelet transform of one dimensional signal $f(x)$ is defined using

$\mathrm{w}(\mathrm{a}, \mathrm{b})=\int f(x) ¥_{\mathrm{a}, \mathrm{b}}(\mathrm{x}) \mathrm{dx}$

where $\quad ¥_{\mathrm{a}, \mathrm{b}}(\mathrm{x}) \mathrm{dx}=\frac{1}{\sqrt{a}} ¥ \frac{(x-a)}{b}$

The wavelet transformation satisfies the localized zero mean function. The above equation can be normalized by restraining ' $a$ ' and ' $b$ ' to a discrete lattice $(a=2 n, b)$. Various few constraints are imposed on $¥$ to ensure that the transformation is non redundant and constitutes a multiresolution representation of the original signal. Two dimensional transformations is performed by applying a separable filter to image for examine low-pass and high pass filters.

The obtained convolution with the low-pass filter process in approximation image and convolution with high pass filter process in detailed feature extracted images. The signal decomposition for an n-level,

$$
\begin{aligned}
& \mathrm{A}_{\mathrm{n}}=\left[\mathrm{Hx} *\left[\mathrm{Hy} * \mathrm{~A}_{\mathrm{n}}-1\right] \downarrow 2,1 \downarrow 2,1\right. \\
& \mathrm{D}_{\mathrm{n} 1}=\left[\mathrm{Hx} *\left[\mathrm{~Gy} * \mathrm{~A}_{\mathrm{n}}-1\right] \downarrow 2,1\right] \downarrow 2,1 \\
& \mathrm{D}_{\mathrm{n} 2}=\left[\mathrm{Gx} *\left[\mathrm{Hy} * \mathrm{~A}_{\mathrm{n}}-1\right] \downarrow 2,1\right] \downarrow 2,1 \\
& \mathrm{D}_{\mathrm{n} 3}=\left[\mathrm{Gx} *\left[\mathrm{~Gy} * \mathrm{~A}_{\mathrm{n}}-1\right] \mid 2,1\right] \downarrow \mid 2,1
\end{aligned}
$$

Where '*' denotes the convolution operator 2,1 denotes the down sampling along the rows and columns $\mathrm{A} 0=\mathrm{I}$ is denoted as the original Image, and $\mathrm{H}$ denotes Low pass filters and $\mathrm{G}$ denotes High pass filters where $\mathrm{I}(\mathrm{x}, \mathrm{y})$ is the original image. An is obtained by low pass filter with the approximation image at scale $\mathrm{n}$. Dni is termed as the detailed image which is obtained by band pass filtering in a specific direction, where $\mathrm{i}=1,2,3$ for vertical, horizontal and diagonal directions at scale $n$. the set of sub-images at several scales $\{A n, D n i\}$ is represented from the original image. 


\section{IMAGE CLASSIFICATION TECHNIQUE}

Pixels are the smallest element represented in an image. Classification of Image uses reflectance statistics for individual pixels.

\section{(i) Supervised technique \\ (ii) Unsupervised technique}

In unsupervised technique classification is termed as the grouping of pixels with common characteristics without providing sample classes. In supervised classification the training sites are given as references for the classification of all other pixels in the image. In which training sites are termed as testing sets or input classes. The user predefines, the set of bounds for similar other pixels must be group together.

The predefined bounds are often set based on the training area, based on feature parameters which are obtained from FOS, GLCM with wavelet transformation. From these results, the classification of landscape classes can be obtained with the help of supervised and unsupervised technique. From the test satellite Image 20x20 sized windows, where segmented using first level and second level features where extracted.

The extracted features were used for training in classifier techniques of supervised and unsupervised techniques. The classifier which classifies the window as provided classification on five land cover regions. The classification of five land cover regions was implemented with the simulation tool MATLAB.

\section{EXPERIMENTAL RESULTS}

\section{Step 1: Preprocessing}

The test satellite image is preprocessed and the features in the image is extracted with the help of first level and second level feature extraction.

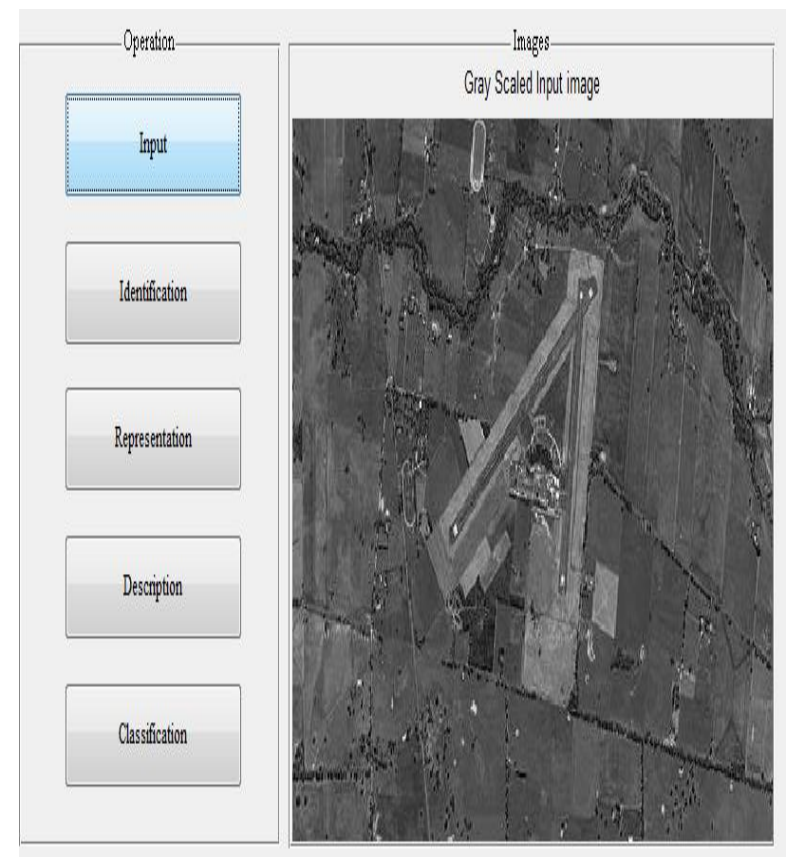

Figure 2: Test satellite image is preprocessing

\section{Step 2: Supervised Classification}

The extracted features where given in classifier for classification. The below diagram represents supervised classification.
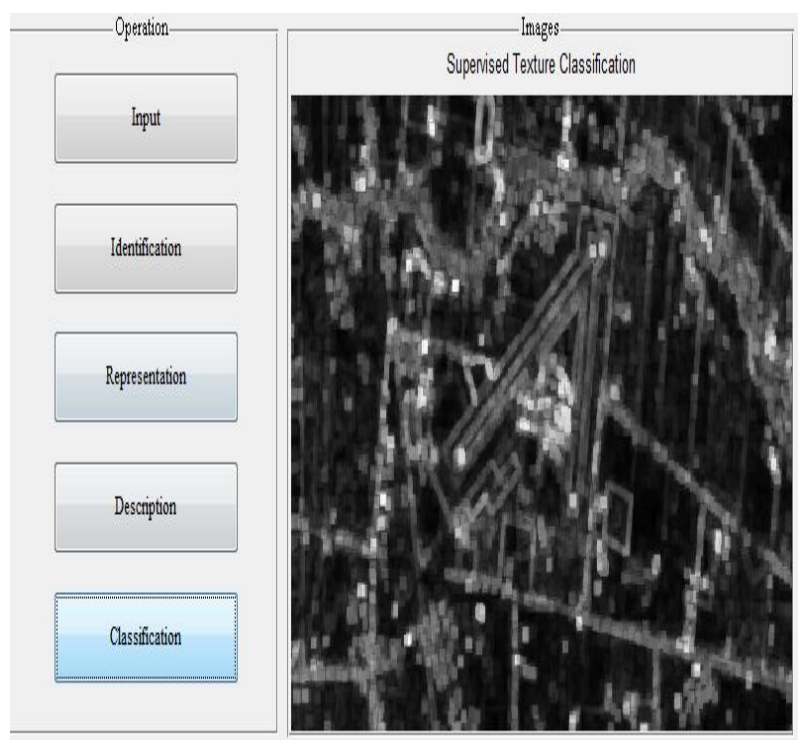

Figure 3: supervised classification in texture pattern.

\section{Step 3: Unsupervised Classification}

The extracted features in classifier represent unsupervised classification.

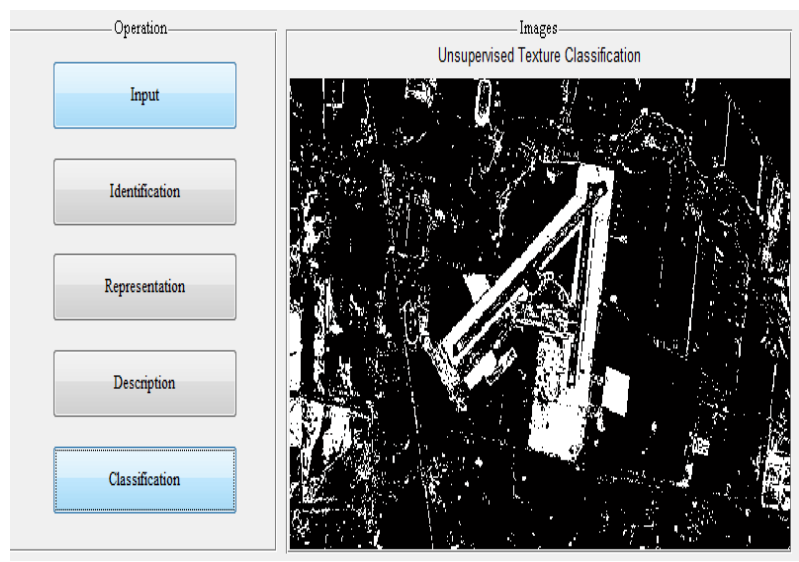

Figure 4: unsupervised classification in texture pattern

Table 1

\begin{tabular}{|l|l|}
\hline Land classes using Classifier & Results in \% \\
\hline Building & 98 \\
\hline Mountain & 96 \\
\hline Vegetation & 96 \\
\hline Water & 96 \\
\hline
\end{tabular}

The table shows classification rates using proposed technique and produces better results when compare to the existing system.

\section{CONCLUSION}

This paper projects the classification of land cover area from the test satellite Image using first order statistics and Gray level Co-occurrence Matrix with wavelet transformation. The 
classification progress has been carried out by supervised and unsupervised classification technique. The proposed scheme of wavelet transformation provides an effective mapping of land cover analysis for remote sensing.

\section{REFERENCES}

[1] D. Menaka, L. Padma Suresh and S.Selvin Premkumar (2015) Wavelet Transform-Based Land Cover Classification of Satellite Images, Artificial Intelligence and Evolutionary Algorithms in Engineering Systems and Computing.

[2] S.Zang, Q.Zhou, (2012) New feature extraction algorithm for satellite image-non-linear small objects, in IEEE Symposium on Electrical and Electronics Engineering

[3] S.Arivazhagan, L.Ganesan (2003) Texture classification using wavelet Transform, Pattern recognition.
[4] A.Baradi, F. Parmiggiani, An investigation of the textural characteristics associated with gray level co occurrence matrix statistical parameters, IEEE Trans.Geosci.Remote Sens.33 (2)

[5] B. Sırmaçek, C. Ünsalan, A probabilistic framework to detect buildings in aerial and satellite images. IEEE Trans. Geosci. Remote Sens. 49(1), 211-221 (2011)

[6] B. Tian, M.R. Azimi-Sadjadi, T.H. Vonder Haar, D. Reinke, Temporal Updating Scheme for Probabilistic Neural Network with Application to Satellite Cloud Classification. IEEE Trans.Neural Networks 11(4), 903$920(2000)$

[7] L.A. Ruiz, A. Fdez-Sarría, J.A. Recio, Texture feature extraction for classification of remote sensing data using wavelet decomposition: a comp study. Remote Sens. Spatial Inf. Sci., in Proceedings of International Archives Photogramm (2004), pp. 1109-1115 